\title{
COMMENTARY
}

\section{Preboarding, Orientation, and Onboarding of New Pharmacy Faculty During a Global Pandemic}

\author{
Kajal Bhakta, PharmD ${ }^{\mathrm{a}}$, Melissa S. Medina, EdD ${ }^{\mathrm{b}, \mathrm{c}}$ \\ ${ }^{a}$ University of Texas at Austin, College of Pharmacy, Austin, Texas \\ ${ }^{\mathrm{b}}$ The University of Oklahoma, College of Pharmacy, Oklahoma City, Oklahoma \\ ${ }^{\mathrm{c}}$ Associate Editor, American Journal of Pharmaceutical Education, Arlington, Virginia
}

Submitted December 10, 2020; accepted December 15, 2020; published March 2021.

Keywords: COVID-19, faculty, tripartite mission, mentoring, career development

\section{INTRODUCTION}

Recruitment of new faculty requires a significant investment of time, effort, and money, so it is important to set up new faculty for short- and long-term success in their new role. ${ }^{1}$ One way to do this is to have new faculty participate in a formal onboarding process as it can increase the retention rate among new employees by $69 \%$. ${ }^{1,2}$ In addition to increasing retention, a formal onboarding program can help new hires reach productivity two months earlier than nonparticipants. ${ }^{3,4}$ This is an important outcome as the first 90 days are a crucial time period for new hires to become functional, engaged, integrated, focused, and productive. ${ }^{5}$

Onboarding is the process of assimilating new employees into an organization, including facilitating relationship formation; clarifying expectations, roles, priorities, and metrics; showing how to access resources; reviewing the organizations' culture, goals, mission, and vision; and providing development opportunities. ${ }^{3}$ The process begins on a new employees' first day. Because of the gap between the hire and start date in jobs like academia, onboarding programs should include pre-employment onboarding (preboarding) as well. ${ }^{1,3}$

Orientation is another part of onboarding new pharmacy faculty and is concerned with the administrative aspects of a new job, such as setting up payroll, parking permits, benefits, ID badges, credentialing, and computer access. ${ }^{3}$ Orientation is an important part of onboarding as it sets the tone for the new employee's transition and shapes their impression of and relationship to the organization. ${ }^{3}$ Overall, successful onboarding and orientation

Corresponding Author: Melissa S. Medina, University of Oklahoma, College of Pharmacy, PO Box 26901, 1110 N.

Stonewall Ave., CPB 225, Oklahoma City, OK 73190. Tel: 405-271-6484. Email: melissa-medina@ouhsc.edu programs can lead to increased satisfaction and retention of new faculty. ${ }^{1}$

Under ordinary conditions it is important for a school to have a formal onboarding process in place, but during a global pandemic it is crucial. Schools and colleges should evaluate their existing onboarding and orientation programs to identify areas where improvement is needed. They should also consider including a structured preboarding process to better connect new faculty, especially those that interviewed virtually during the pandemic, as they may have missed the interpersonal communication that occurs in an in-person interview. Programs should also consider formally assigning a mentor for each area (preboarding, orientation, and onboarding) to better guide their new faculty since the opportunity to meet new colleagues and acclimate to a new environment are decreased due to quarantining and social distancing during the pandemic. Failure to explicitly identify these three mentors for new faculty can lead everyone to assume that someone else is doing it, which can result in a lack of intentional support and guidance for the new faculty member. The department chair is instrumental in the process of identifying potential mentors and arranging training, as mentor training is becoming more available. ${ }^{6}$ The mentors that are identified should be available, engaged, and interested in investing in guiding, supporting, and developing new faculty. ${ }^{7}$ They should be knowledgeable enough to point new faculty to the right resources and connected enough to serve as a bridge to teaching, research, or service collaborations. Relatedly, the mentee (ie, the new faculty member) should be openminded and receptive to input, reflective about and assertive in identifying their needs, and committed to communicating. The following sections offer recommendations for the specific role mentors can serve and adjustments that could be made for the preboarding, orientation, and onboarding for new faculty as a result of COVID-19. 


\section{American Journal of Pharmaceutical Education 2021; 85 (3) Article 8510.}

\section{DISCUSSION}

\section{Preboarding Mentors During COVID-19}

For faculty candidates seeking a job during COVID19 , recruitment has most likely looked very different than prior to the pandemic, with Zoom interviews held in lieu of in-person interviews. The absence of an in-person first and even second interview can be challenging for faculty candidates as they may be missing details that facilitate accepting a new job offer, such as exploring the area, looking at real estate, eating out with faculty hosts, and interacting with the department and students. If a faculty candidate accepts an offer, especially an out-of-state position, the department chair should identify a preboarding mentor as soon as possible to help fill the void left from a virtual interview process. Assigning a preboarding mentor can be helpful even for a person who completed an inperson interview, especially if they are relocating to a new area without an existing social or familial network, as the need for support and guidance is high during this transition time.

To facilitate a good mentor fit, the new faculty member (ie, mentee) should identify important moving and relocation priorities and questions. For example, if the new faculty member has school-aged children, a mentor with children may be a good match because they could discuss possible communities for the new hire to live in and desirable school districts in the area. While some new faculty may want to move early to unpack and explore their new town, relocating during COVID-19 may require quarantining upon arrival and adjusting timelines. Because of social distancing and decreased gatherings, new faculty may have limited opportunities to meet new people in their community. The preboarding mentor could suggest alternate ways to explore the new town and engage with the community, such as recommending popular restaurants with take-out dining options, safe outdoor adventures, scenic drives, and drive-in movie theaters. Overall, while welcoming new faculty during a pandemic can present challenges, a preboarding mentor can be an excellent resource during this acclimation period.

\section{Orientation for Mentors During COVID-19}

During the pandemic, it is crucial for department chairs to explicitly identify who is serving as the orientation mentor for the new faculty member as many orientation requirements may have changed. This formal mentor may be the department secretary or human resources manager as they typically serve in this role and are knowledgeable about orientation. For example, the orientation mentor can help a new faculty member who is moving from another state to apply to take state licensure examinations. This assistance is important as it is vital for the new faculty member to sign up as early as possible in order to be licensed in time to start their new job. The orientation mentor can also assist new faculty on their first day of work by helping them to complete required paperwork (eg, benefits, timesheets, payroll, and HR training); acquire an employee ID badge; gain office, building, and practice site keys or access cards; identify internet technology (IT) support for secure, encrypted, WiFi and on-and-off campus network connections; access library and virtual private networks (VPNs); secure computer passwords, equipment, supplies, and parking permits; and obtain and set up an office. ${ }^{8}$

The orientation mentor can also help new faculty with completing credentialing paperwork necessary to acquire practice site privileges. The practice site, university, and each department may need to have separate orientation sessions, so the orientation mentor can notify new faculty of these dates and explain how to attend each orientation session, whether it is live, recorded, or virtually delivered. Helping new faculty schedule and attend orientation sessions is important because there may be a limited number of orientation dates available and failure to attend an orientation session may interfere with a faculty member getting credentialed in a timely fashion.

During the pandemic, the department chair may also want to consider identifying a second orientation mentor to serve as an additional resource for new faculty beyond the assistance described above. This additional orientation mentor's role could be more informal, such as welcoming the new faculty member, helping to answer questions, and serving as a friendly face to have lunch or coffee with, which may be vitally important if few people are working in the building and most are working from home.

\section{Onboarding Mentors During COVID-19}

Traditionally, identifying a mentor for career development can be highly beneficial and may result in higher career satisfaction. ${ }^{9,10}$ Having an onboarding mentor is an important first step in career development. Prior to COVID-19, faculty and staff may have been easily accessible to new faculty to answer questions. Now, many colleagues may be working from home, and a new faculty member may be unsure of who to ask for help. An onboarding mentor can serve as a "sponsor" whose job it is to facilitate introductions by connecting, advocating, and using their influence to make recommendations. ${ }^{11}$ Onboarding mentors should serve as a bridge for early relationship formation and formally introduce the new faculty member to faculty, staff, and students to decrease the guesswork behind "the new person" in the building. The onboarding mentor can augment these introductions 


\section{American Journal of Pharmaceutical Education 2021; 85 (3) Article 8510.}

by suggesting or arranging meetings with specific faculty, committee chairs, and administrators to help him/her get to know the college and/or department and its specialties. Traditionally, these introductions could be accomplished through informal lunches or coffee, but now may be replaced with virtual meetings. The orientation mentor can set up virtual happy hours with the department or individual faculty members, which can be a great way to cultivate a sense of community, camaraderie, and understanding for the new faculty member. ${ }^{12}$ By doing this, the onboarding mentor can encourage others to invest in the new faculty member, make them feel welcomed, and promote future collaborations.

Furthermore, with the pandemic, it may be difficult for incoming faculty to learn the expectations and culture of the college, especially related to working during COVID-19. The onboarding mentor can serve as a guide whose job it is to explain how things are done at the school, help new faculty find their way, and learn the institutional history. ${ }^{11}$ As a guide, the onboarding mentor can give valuable insight into the school's pre-COVID-19 work environment and expectations and how it may have changed. For example, the mentor can explain if workfrom-home and one-on-one virtual meetings are encouraged or if the culture is for the faculty member to be present in their office and make attempts to physically distance when meeting with others. The mentor can also highlight essential department, college, and university meetings to attend, when and where they are held, and which meetings are optional. Onboarding mentors can provide the new faculty member with an understanding for what is accepted and expected, as well as what to expect from others.

The onboarding mentor should advocate new faculty members to self-reflect and assess areas in which they would like more assistance and training. For example, when starting work at a practice site, new faculty could observe other clinical pharmacists who are performing similar tasks, have discussions with administration about practice site goals to focus on and timelines, and identify expectations for both the practice site and the school. The onboarding mentor may also be able to help the new faculty identify additional mentors in the school or on campus. Onboarding mentors, with oversight from the department chair, may even help the new faculty member with setting feasible goals to achieve throughout their first year. This is an important task especially during the pandemic, as some goals may not be as easily achievable as they were in the past. For example, faculty members may need to delay pursuing promotion because of COVID-19. New faculty may have to alter their timelines for precepting; establishing a practice site; conducting face-to-face patient visits; conducting research; obtaining national grants; building a local, regional, or national presence; or giving presentations at local or national meetings.

One additional role that the onboarding mentor could serve is that of an active listener. As an active listener, they can ask the new faculty how things are going and they can listen, advise, relate, support, and encourage. ${ }^{11}$ Starting a new job is a major life stressor and one that has been compounded by COVID-19. ${ }^{13,14}$ Being a good listener does not mean that the mentor should be a counselor or problem-solver. However, the mentor can solicit the new faculty member's worries and apprehensions, guide them to resources, encourage them to be proactive and assertive about their needs and concerns, and advocate that they begin seeking additional mentorship for each of the aspects of the tripartite mission, with a focus on promotion and/or tenure. The mentor can recommend follow-up meetings with specific individuals. They can also reassure the new faculty member if frustrations or barriers arise.

\section{CONCLUSION}

A new faculty member's reality during COVID-19 may include making extra effort to meet with people because of closed doors and personnel working off-site, limited help from on-site staff, help and closed resource offices (eg, IT, notaries, financial, etc). Asking for help is not as simple as walking to the office next door. Now it may require navigating proper channels and sending multiple emails to get an answer to a simple question. It is also more difficult to get to know people, as in order to do so, one would likely have to set up a meeting. This puts a strain on new faculty as they are in their own silos, and it is difficult for them to grasp what their roles and responsibilities are, what is mandatory vs what is optional, and what is frowned upon vs what would be appreciated. Because human connections are harder to make, it is even more difficult for new faculty to find and establish new mentors.

Most colleges make significant investments to recruit and hire new faculty, but during the pandemic, once a new faculty is hired, a structured and intentional preboarding, orientation, and onboarding program may be lacking, and new faculty may not be assigned mentors to specifically address this early timeframe. Instead, mentors may be assigned after faculty get settled or the focus is on promotion and tenure or the tripartite mission specifically. Now more than ever, new faculty can benefit from preboarding, orientation, and onboarding mentors who serve as bridges and ambassadors to all aspects of the university. Without them, new faculty may get lost in the 


\section{American Journal of Pharmaceutical Education 2021; 85 (3) Article 8510.}

competing priorities that universities are facing during the pandemic, which can alter their successful transition to their new job as well as the trajectory of their early career.

\section{REFERENCES}

1. Baker B, DiPiro JT. Evaluation of a structured onboarding process and tool for faculty members in a school of pharmacy. Am J Pharm Educ. 2019;83(6):Article 7100.

2. Lynch K, Buckner-Hayden G. Reducing the new employee learning curve to improve productivity. J Health Risk Manag. 2010;29:22-28. 3. Ross WE, Huang KH, Jones GH. Executive onboarding: ensuring the success of the newly hired department chair. Acad Med. 2014;89: 728-733.

4. Ganzel R. Putting out the welcome mat. Training. 1984;35(3): 54-63.

5. Watkins MD. The First 90 Days: Proven Strategies for Getting Up to Speed Faster and Smarter. Updated and Expanded. Boston, MA: Harvard Business School Press. 2013:15-20.

6. DeRosa $\mathrm{D}$. Why mentoring during the onboarding process can be beneficial. September 30, 2019. https://www.business2community. $\mathrm{com} /$ strategy/why-mentoring-during-the-onboarding-process-isbeneficial-02244200\#: :text $=$ While $\% 20$ mentoring $\% 20$ programs $\%$ 20are $\% 20$ often,culture $\% 20$ and $\% 20$ expectations $\% 20$ first $\% 20$ hand. Accessed March 5, 2021.

7. Leiberman D. How to select a mentor as a trainee and junior faculty. Gastroenterology. 2016;151(1):17-19.

8. Whitaker M. How to start off right in your new job. Chronicle of Higher Education. July 16, 2019. https://www.chronicle.com/article/ how-to-start-off-right-in-your-new-job/. Accessed March 5, 2021. 9. Sambunjak D, Straus S, Marusic A. Mentoring in academic medicine: a systematic review. JAMA. 2006;296(9):1103-1115. 10. Feldman M, Arean P, Marshall S, Lovett M, O’Sullivan P. Does mentoring matter: results from a survey of faculty mentees at a large health sciences university. Med Educ Online. 2010;15:5063.

11. Sandberg S. Lean In: Women, Work, and the Will to Lead. New York, NY: Random House. 2013.

12. Maurer R. Virtual happy hours help co-workers, industry peers stay connected. SHRM. April 6, 2020. https://www.shrm.org/hrtoday/news/hr-news/pages/virtual-happy-hours-help-coworkersstay-connected.aspx. Accessed March 5, 2021.

13. Holmes TH, Rahe TH. The social readjustment rating scale. $J$ Psychosomatic Res. 1967;11:213.

14. Mayers A. Ten most stressful life events and ways to handle them. Thrive Global. 4May, 2018. https://thriveglobal.com/stories/ 10-most-stressful-life-events/. Accessed March 5, 2021. 